\title{
Numerical and experimental diagnosis of complex rotor system by time-frequency techniques
}

\author{
Bernard Xavier Tchomeni ${ }^{\mathrm{a}}$ and Alfayo Alugongo \\ Department of Mechanical Engineering, Vaal University of Technology, 1911, South-Africa.
}

\begin{abstract}
This paper describes the application of Discrete Wavelet Transform (DWT) to identify various types of nonlinear damage caused by, unbalance, rotor-stator contact and a breathing crack in rotating machinery. Multiple faults have been investigated based on numerical and experimental signal analysis using Fast Fourier Transform (FFT) and DWT. A four degree of freedom fully coupled model of the rotor-stator system that includes the nonlinear damage in the rotor vibrations was established using Energy principles. Existence of high system nonlinearity could not allow exhaustive discrimination of rub and crack by classical FFT. Therefore, the DWT was employed. The results provide detailed feature analysis of the fault signals. Practical vibration measurements through a data acquisition system interfaced with Rotor Kit-4 and crack simulator provided the test data. Experimental TimeFrequency analysis gave more realistic faults responses with variable faults features. Irregularity of orbit, harmonic peaks in the presence of rub and crack were unique and distinguished periodic motion from other types of motion. The presence of a crack shifted the critical speed location and exhibited sub-harmonic components, which were more prominent with rub in vibration response. The detailed decomposition signal by DWT method established inherent feature patterns that effectively discriminated the multiple faults.
\end{abstract}

\section{Introduction}

Rotating shafts play an important role in many sectors of industry, and their dynamic behaviour and fault diagnosis have a significant economic importance. Thus, the risk of unbalance, rub-impact and cracks is an important form of rotor damage that can lead to disastrous failures if undetected in time. Therefore, it is imperative for fault analysis of the rotating system to extract significant features from time-domain vibration response of various faults combinations. Rotors are relatively more flexible and operate under harsh and hostile environmental conditions. Thus, condition monitoring of rotor system becomes more complicated, especially when faults existing in the system are more than one.

Rotor systems may harbour unbalance, undergo rub contact with a stationary part under tighter clearances or could develop fatigue crack under severe thermal and mechanical stresses [1], amounting to a coexistence of multiple faults. Several researchers have used various techniques to diagnose a crack and rotor-stator rub under exciting centrifugal force.

The cracked rotor has been analysed experimentally to investigate nonlinear dynamic characters of rotor system containing a transverse fatigue crack [2]. Results from this analysis revealed from the

\footnotetext{
${ }^{\mathrm{a}}$ Corresponding author : bernardt@vut.ac.za

(C) The Authors, published by EDP Sciences. This is an open access article distributed under the terms of the Creative Commons Attribution License 4.0 (http://creativecommons.org/licenses/by/4.0/).
} 
orbit and spectrum of the rotor's centre that, the effect of the crack becomes obvious only if the crack is deep enough. Based on the model-based diagnostic approach, [3] studied multiple faults identification of rotor system by a least-squares fitting approach in the frequency domain.

The signal-based approach for vibration analysis of the rotor system with combined crack and asymmetry faults was performed in [4]. Both the Jeffcott rotor model and Timoshenko beam element were used to numerically and experimentally investigate the rotor vibration characteristics of the unbalance, crack, and rotor-stator rub faults [5]. Recently, several authors have shown interest in new techniques of data analysis such as the Wigner-Ville distribution for analysis of linear but nonstationary signal $[6,19,20]$. Furthermore, various nonlinear time-frequency transformations methods such as Wavelet Transform (WT) were designed for non-stationary and nonlinear systems. The principle of WT and their numerical applications in signal processing have been widely used to simulate the transient response of an accelerated cracked Jeffcott rotor with a switching crack and investigated the detection and monitoring of cracks in a rotor-bearing system [7-10].

Literature review indicates that WT technique has been successfully used to simulate various faults and to perform damage localization. [11] presented an experimental study of the dynamic response of an overhang rotor with a propagating transverse crack using the Discrete Wavelet Transform (DWT). [12] showed the efficacy of WT in detecting transient waves in a dispersive medium by applying WT to the experimental analysis of the transient vibrations of an impact excited uniform beam.

Mostly, previous literature in multiple fault analysis is purely simulation and the need of validating experimentally the different numerical proposed method is requested.

Therefore, this paper presents both a numerical and experimental study on the dynamic response of a rotor with multiple faults namely unbalance, rub-impact and transverse crack using Fast Fourier Transform and the Discrete Wavelet Transform (DWT). Start-up and steady-state vibration signatures have been investigated using Daubechies $(\mathrm{Db} n)$ mother wavelet. Results are discussed and a number of observations on the rotor-stator system are highlighted in this paper. The basic system layout used is shown in figs 1 through 2 .

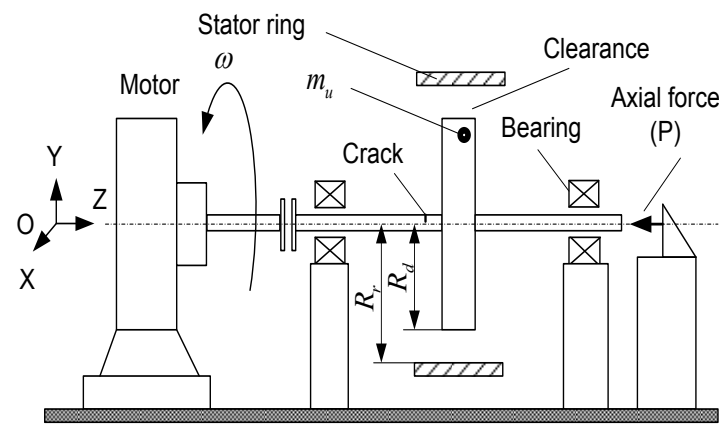

Figure 1. Motor with shaft-disc-stator-transverse crack

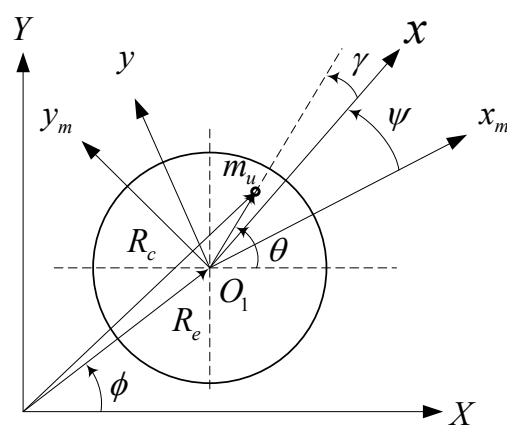

Figure 2. Section view of the disc the shaft

\section{Model of Study and Numerical Simulation}

Based on the geometry of Figs 1 to 2, and considering modal damping, the kinetic energy, potential energy, and Rayleigh's dissipation function of the motor-disc-shaft system are gave below [13]:

$$
\begin{gathered}
U_{D}=\frac{1}{2} M\left(\dot{X}^{2}+\dot{Y}^{2}\right)+\frac{1}{2} J_{D}\left(\dot{\theta}_{1}+\dot{\psi}_{1}\right)^{2}+\frac{1}{2} J_{M} \dot{\theta}^{2}+\frac{1}{2} m_{u} \dot{R}_{e}^{T} \dot{R}_{e} \\
V=\frac{1}{2} K_{X X} X^{2}+\frac{1}{2} K_{Y Y} Y^{2}+\frac{1}{2} K_{\psi \psi} \psi^{2}
\end{gathered}
$$




$$
D=\frac{1}{2} C_{X X} \dot{X}^{2}+\frac{1}{2} C_{Y Y} \dot{Y}^{2}+\frac{1}{2} C_{\psi \psi} \dot{\psi}_{1}^{2}
$$

where, $m_{u} J_{D}, J_{M}, \theta, \psi$ and $\dot{R}_{\mathrm{e}}$ respectively are, unbalance mass, disc-mass moment of inertia, rotormass moment of inertia, rigid-body rotation, twist about Z-axis and velocity vector of $m_{u}, K_{X X}, K_{Y Y}$, and $K_{\psi \psi}$ are the stiffness coefficients associated with the system degrees of freedom, and $C_{X X}, C_{Y Y}$, and $C \psi \psi$ are the respective damping coefficients. A high rotor speed excites axial and lateral vibrations which in turn excites an axial force and torque that affects bending stiffness as follows [14]:

$$
K_{X X}=K_{Y Y}=K_{b}-\frac{P \pi^{2}}{2 L}-\frac{F_{\psi} \pi^{3}}{2 L^{2}}
$$

where, $K_{b}, P$ and $F_{\psi}$ correspondingly are, modal stiffness for first mode bending, fluctuating axial force and axial torque on the rotor. In this paper, $P$ and $F_{\psi}$ are defined as follows:

$$
P=P_{0}+P_{f} \sin \theta, \quad F_{\psi}=T_{0}+T_{f} \sin \theta
$$

where, $P_{f}, T_{f}$ and $\theta$ are amplitudes and rigid-body rotation angle respectively. In this paper $P$ and $F_{\psi}$ are prescribed a priori as in [13]:

$$
P=0, F_{\psi}=0
$$

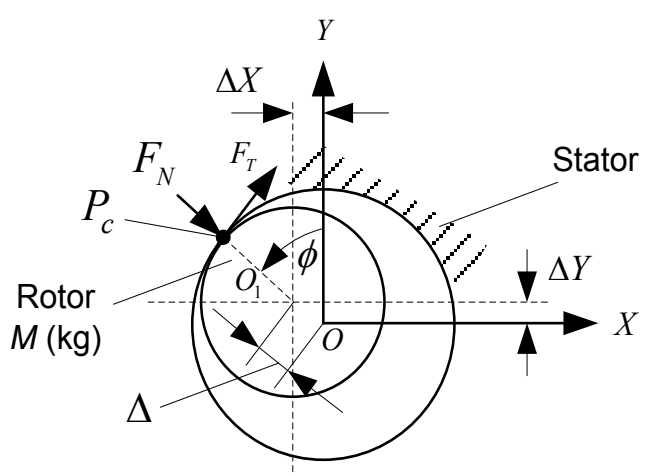

Figure 3. Geometry of rotor-stator forces and a clearance.

Applying the approach in [13], to Fig. 3, the position of the shaft center and the rub-impact forces in Cartesian coordinate frame are:

$$
\begin{aligned}
& F_{X}=-F_{N} \cos \phi+F_{T} \sin \phi \\
& F_{X}=-F_{X} \sin \phi-F_{T} \cos \phi
\end{aligned}
$$

where, $F_{N}, F_{T}$ respectively are, radial component of rub force, tangential component of rub force. The lateral forces in $X$ and $Y$ directions are [13]:

$$
\begin{aligned}
& F_{X}=-K_{S}\left(1-\frac{\Delta}{R}\right) X+K_{S}\left(1-\frac{\Delta}{R}\right) \mu Y \\
& F_{Y}=-K_{s}\left(1-\frac{\Delta}{R}\right) \mu X-K_{s}\left(1-\frac{\Delta}{R}\right) Y
\end{aligned}
$$

where, $\mu$ and $K_{S}$ respectively are coefficient of dry friction and stator radial stiffness. 
Subsequently, a hinge mechanism in [10] was adopted in the shaft to provide for local flexibility effect of a breathing crack. Ignoring shear stresses, observing Saint-Venant's principle and following the approach in [16], the cracked rotor stiffness is given as,

$$
K_{0}+\Delta k(\theta)=\left[\begin{array}{cc}
k_{x x} & 0 \\
0 & k_{y y}
\end{array}\right]-\frac{1}{2} f(\theta) \Delta k_{x x}\left[\begin{array}{cc}
1-\cos 2 \theta & \sin 2 \theta \\
\sin 2 \theta & 1+\cos 2 \theta
\end{array}\right]
$$

where, $\Delta k_{x x}, K_{0}$ and $f(\theta)$ respectively, are change in stiffness of the shaft in the weaker axis due to the crack, uncracked shaft stiffness and steering function $f(\theta)$ as indicated in [16].

Considering Eqs. (6) and (7) the dynamic equation of the unbalanced-cracked-rub rotor-stator system is:

$$
\begin{aligned}
& {\left[\begin{array}{cccc}
m_{\theta \theta} & m_{\theta \psi} & m_{\theta X} & m_{\theta Y} \\
m_{\psi \theta} & m_{\psi \psi} & m_{\psi X} & m_{\psi Y} \\
m_{X \theta} & m_{X \psi} & m_{X X} & 0 \\
m_{Y \theta} & m_{Y \psi} & 0 & m_{Y Y}
\end{array}\right]\left\{\begin{array}{c}
\ddot{\theta} \\
\ddot{\psi} \\
\ddot{X} \\
\ddot{Y}
\end{array}\right\}+\left[\begin{array}{cccc}
0 & 0 & 0 & 0 \\
0 & C_{\psi \psi} & 0 & 0 \\
0 & 0 & C_{X X} & C_{X Y} \\
0 & 0 & C_{Y X} & C_{Y Y}
\end{array}\right]\left\{\begin{array}{c}
\dot{\theta} \\
\dot{\psi} \\
\dot{X} \\
\dot{Y}
\end{array}\right\}+\left[\begin{array}{cccc}
0 & 0 & 0 & 0 \\
0 & K_{\psi \psi} & 0 & 0 \\
0 & 0 & K_{X X} & K_{X Y} \\
0 & 0 & K_{Y X} & K_{Y Y}
\end{array}\right]} \\
& \left\{\begin{array}{c}
\theta \\
\psi \\
X \\
Y
\end{array}\right\}+\left\{\begin{array}{c}
Q_{\theta} \\
0 \\
Q_{X} \\
Q_{Y}
\end{array}\right\}+\frac{1}{2} f(\theta) \Delta k_{x x}\left[\begin{array}{cccc}
0 & 0 & 0 & 0 \\
0 & 0 & 0 & 0 \\
0 & 0 & 1-\cos 2 \theta & \sin 2 \theta \\
0 & 0 & \sin 2 \theta & 1+\cos 2 \theta
\end{array}\right]\left\{\begin{array}{c}
0 \\
0 \\
0 \\
Y_{S}
\end{array}\right\}=\left\{\begin{array}{l}
F_{\theta} \\
F_{\psi} \\
F_{X} \\
F_{Y}
\end{array}\right\}
\end{aligned}
$$

\section{A brief description on WT and DWT}

WT is a signal processing tool and decomposes a nonstationary signal into scales (also known as levels) with different time and frequency resolutions. It is probably the most recent solution to overcome the shortcomings of the FFT. As opposed to FFT which breaks up a signal into sine waves of different frequencies, WT breaks up a signal into shifted and scaled versions of the original (mother) wavelet [17]. For nonstationary signals, the Fourier analysis is not effective since it transforms the signal into the frequency domain and the time information gets lost. WT analysis, allows a windowing technique with variable-sized regions, and permits the use of a time adaptive interval to analyse high and low frequency components of a signal [17]. Furthermore, researches have indicated that, as compared to FFT, WT produces results from which faults are clear to distinguish and easy to interpret [17]. WT can be categorized as Discrete WT or Continuous WT. The CWT time-frequency transform of the initial signal $s(t)$ is expressed as:

$$
C W T_{s}(a, b)=\int s(t) \cdot \psi_{a, b}^{*}(t) d t
$$

where $a$ and $b$ are the, scale parameter and time translation factor respectively. The term, $\psi_{a, b}^{*}(t)$ denotes the complex conjugate of $\psi_{a, b}(t)$. By varying $a$ and $b$, the WT coefficients $W_{s}(a, b)$ can offer the representation of the signal $s(t)$ at different levels of resolution and time shift. In the process, thus, the WT enables one to extract features of the signal $s(t)$. The DWT is derived from the discretization of CWT. It serves as a tool that separates data into various frequency components, and enable to us to study each component with resolution matched to its scale. DWT is computed with a cascade of filtering followed by a factor 2 subsampling [6], and is given by: 


$$
D W T_{s}(j, k)=1 / \sqrt{2^{j}} \int_{-\infty}^{+\infty} s(t) \psi^{*}\left(\left(t-2^{j} k\right) / 2^{j}\right) d t
$$

where $j$ and $k$ are integers, $a$ and $b$ are replaced by $2^{j}$ and $2^{j} k$. By using the DWT the signal $s(t)$ can be analysed at various levels of resolution, known as "multiresolution signal decomposition technique" [17]. In this work DWT has been computed by a successive low and high-pass filtering of the discrete signal $s(k)$, so that the original signal is broken down into many lower resolution components.

\section{Expérimental Setup}

Fig. 4 shows the adopted setup of the RK-4 test rig. The rig is constituted of a motor speed control, four proximity probes and a set of data acquisition interface unit controlled by Ascent toolbox. The piece of equipment designed for a test was set up on a rigid base to avoid disturbances that could influence the measurements. The test rig carried an elastic shaft and one rigid disc driven by a motor shaft through a flexible coupling. The rotor shaft was supported on two roller bearings at both ends and sustained in position by two fixed steel supports (designated as supports A1 and A2 in Fig. 4)

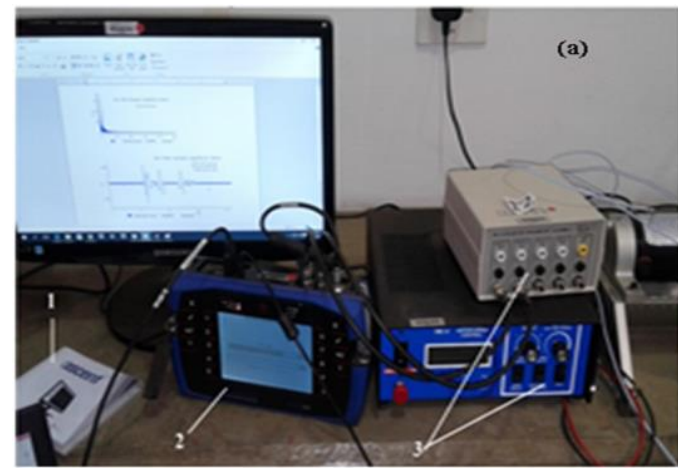

(a) Data acquisition. 1- Ascent; 2-Scout 100; 3-Data Acquisition

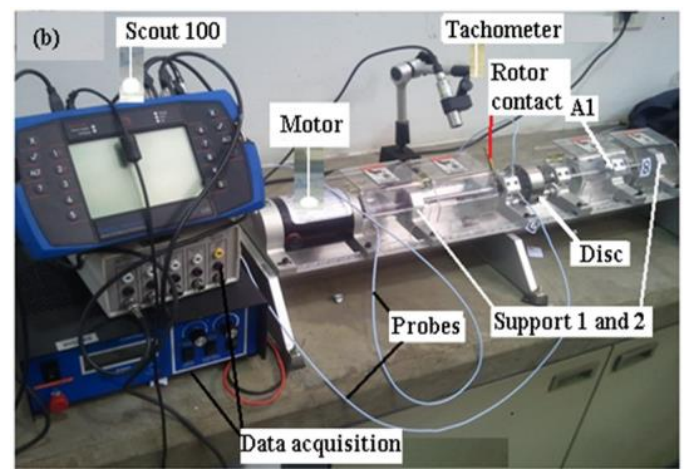

(b) Experimental set up

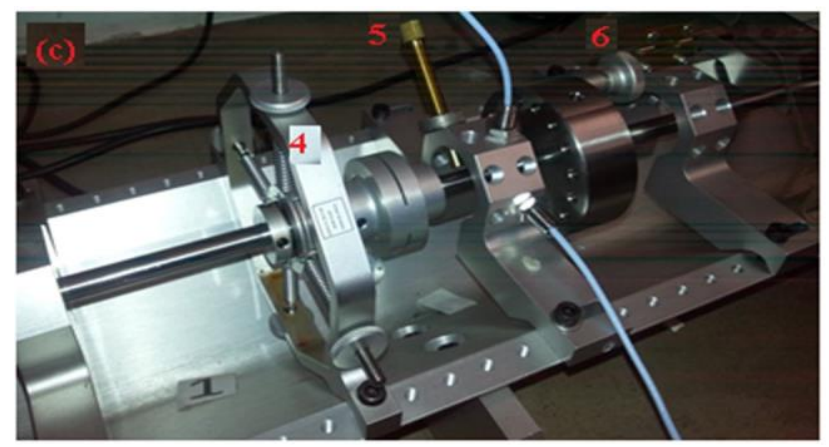

(c) R-K- 4 faults. 4-Crack simulator; 5- Tight screw for rub; 6-Unbalance mass

Figure 4. R-K-4 faults detection test rig with data acquisition

The shaft's acceleration was captured by a laser tachometer. Probes were placed at distances appropriate to detect and measure rotor shaft vibration in various positions. Caution was taken to avoid prospective contact between the probe and a section of the revolving assembly under extreme 
conditions of vibration. All the outputs were integrated with a filter and displayed on a screen of the oscilloscope for further analysis.

Table 1 gives the physical parameters of the RK-4 rig. The signals were recorded in radial direction at various rotating speeds by four proximity probes staggered in the probe block as shown in Fig. 4. The rotor was driven by an AC motor. The maximum rotating speed was set at $5000 \mathrm{rpm}$ for unloading case. For a loading case the rotating speed was fixed at $3000 \mathrm{rpm}$. Two mutually perpendicular proximity probes recorded the relative displacement of the shaft. Two other probes were used for phase reference pulse generation and motor speed control. Rotor-stator rub was realized at point (A1) by introducing a small tight screw at a favourable distance between the rotor and the stator. Motor speed control was set to ensure that, the rotor was driven only in one direction in order to avoid reversal of the friction forces induced by rub.

The data analysis software used for signal's visualization, combined Ascent 2013 linked with a Scout 100 data acquisition board. Scout 100 offered a processing unit that incorporates the capabilities of oscilloscopes, spectrum analysers, filters, recording instruments and directly interfaced with a computer. The acquired data was then subjected to digital processing by FFT and DWT.

Table 1. Rotor system RK- 4 parameters

\begin{tabular}{|l|l|l|l|}
\hline Shaft & Value & Crack & Value \\
\hline Length of the rotor (L) & $570 \mathrm{~mm}$ & From the left bearing & $240 \mathrm{~mm}$ \\
\hline Shaft Diameter & $10 \mathrm{~mm}$ & Depth & $5 \mathrm{~mm}$ \\
\hline Outer Diameter (D) & $75 \mathrm{~mm}$ & Unbalance eccentricity & $10 \mathrm{~mm}$ \\
\hline Inner Diameter (d) & $10 \mathrm{~mm}$ & Motor & \\
\hline Density of the material & $7800 \mathrm{kgm}^{-3}$ & Frequency & $500 \mathrm{~Hz}$ \\
\hline Modulus of elasticity & $211 \times 10^{9} \mathrm{~Pa}$ & Volt & $415 \mathrm{~V}$ \\
\hline Damping coefficient & $0.8 \times 10^{-6} \mathrm{~s}^{-1}$ & Ampere & $1.05 \mathrm{Amp}$ \\
\hline Discs & Value & Discs & Value \\
\hline Mass & $0.845 \mathrm{~kg}$ & Eccentricity mass & $0.018 \mathrm{~kg}$ \\
\hline
\end{tabular}

\section{Simulation, experimental results and discussion}

To investigate vibrations induced by the external forces, the system parameters in [12] have been adopted for simulation. Two investigations were conducted, the first being the numerical simulation of model Eq. (8) on diverse hypothesis by a Modified Runge-Kutta algorithm in MATLAB, giving $X, Y, \theta$ at a time step $\Delta t=0.001 \mathrm{~s}$ in the interval $t=\left[\begin{array}{ll}0 & 20\end{array}\right] \mathrm{s}$. The response at rotor start-up with and without external forces, have been presented and discussed. Subsequently, in a set of experiments, analysis was carried out first in absence of external excitation forces. Vibrations in axial direction did not provide information on rub and crack and therefore its results will not be presented hereafter.

\subsection{Effect of lateral motion and axial force}

In this simulation the torsional deflections $\psi$ was considered to be negligible in comparison to other deflections. The variable stiffness was taken to be equal to the dynamic stiffness and the cracked shaft stiffness considered. Cross-coupling stiffness and damping were neglected $\left(C_{X X}=C_{Y Y}=K_{X X}=K_{Y Y}=0\right)$. Subsequently, a joint examination of frequency spectrum, rotor trajectory, orbit patterns, and DWT, enabled the features of unbalance, rub, and crack combined with rub to be discretized from model and experimental vibration test data. Unbalanced rotor responses at a high speed were obtained numerically by integrating Eq. (8). Figs. 5 and 6 illustrate the obtained results. 


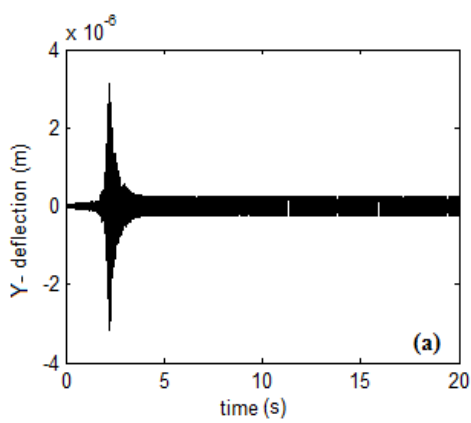

a) Vertical shaft deflection

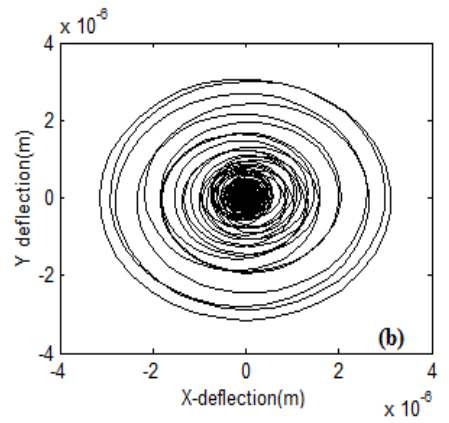

b) Rotor response orbit

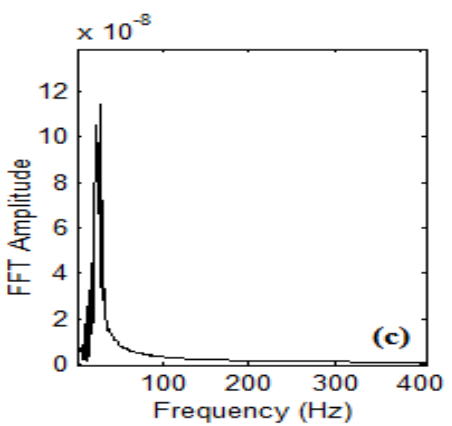

c) Y- frequency rotor signal

Figure 5. Simulated dynamic response of unbalanced shaft, with axial force but no rub, crack, $\Delta K / K_{b}=0$.

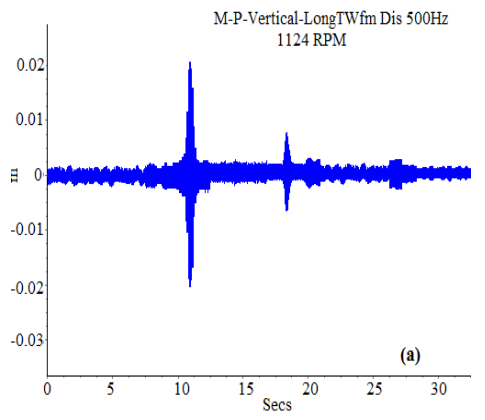

a) Vertical shaft deflection

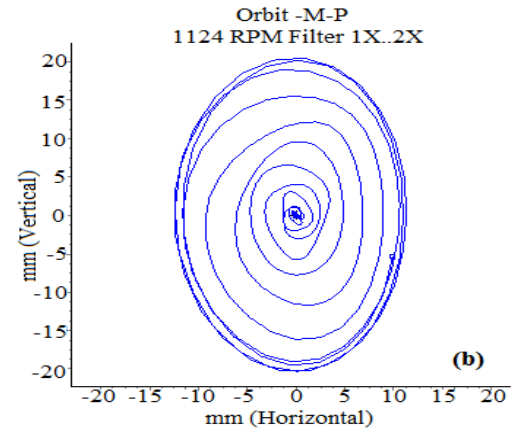

b) Unbalanced rotor response orbit

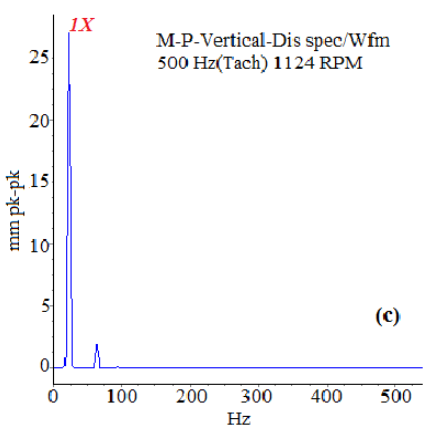

c) Experimental FFT signal

Figure 6. Experimental dynamic rotor system response with unbalance at high speed $2570 \mathrm{rpm}$

Obtained results of unbalance in the absence of a crack and rub are shown in Figs. 5 and 6. Comparing the orbits in Figs. 5 and 6, it was apparent that, the unbalance was a source of extra evolutions in the response, especially when the rotating speed fell near half of the system critical speed. It is noted in Figs. 5 and 6 that, unbalance induced a closed-loop containing a circular loop. As seen in Figs. 5 (b) and 6 (b), the orbit response initially varied from a minimum at the start-up to a maximum at $R=23.3 \mathrm{~mm}$ at resonance and ceded into a regular closed inside loop. The maximum orbit amplitudes in Fig. 5(b) were modified from $R_{1}=3.167 \times 10^{-6} \mathrm{~m}$ to $R_{2}=2.3 \times 10^{-6} \mathrm{~m}$ in Fig. 6(b) when the critical speed changed correspondingly from, $\omega 1=1132.27 \mathrm{rpm}$ in Fig. 5 (c) to $\omega 2=124.43$ rpm in Fig. 6 (c). In Fig. 6 (c), the frequency amplitudes of the experimental data was greater than that of the model data under a small axial force in Fig. 5 (c). The FFT in Fig. 6 (c) reveal that, a high vibrational level was attained by order $1 X$ harmonic at $23.46 \mathrm{~Hz}$. Figs 5 (a) and 6 (a) indicate that, resonance occurred at different times corresponding to the critical speeds, $\omega 1$ and $\omega 2$. Correlation between model and experimental results was perceived in deflection shape, frequencies and orbits.

Further, Daubechies wavelet were adopted for diagnosis of unbalance, rub and crack respectively. Using DWT, a multiresolution analysis of the vibration response of the rotor with and without faults was performed and results illustrated in Figs.7and 8.

To explore the constituent components of the original signal at each level, DWT was performed up to the decomposition level 3 (D3) and approximation coefficients 3 (A3) using Daubechies $\mathrm{db}_{\mathrm{i}}$, $i=[1 \ldots . .7]$ on experimental data. The response was further reconstructed to examine the constituent component of the original signal at each detail level. 


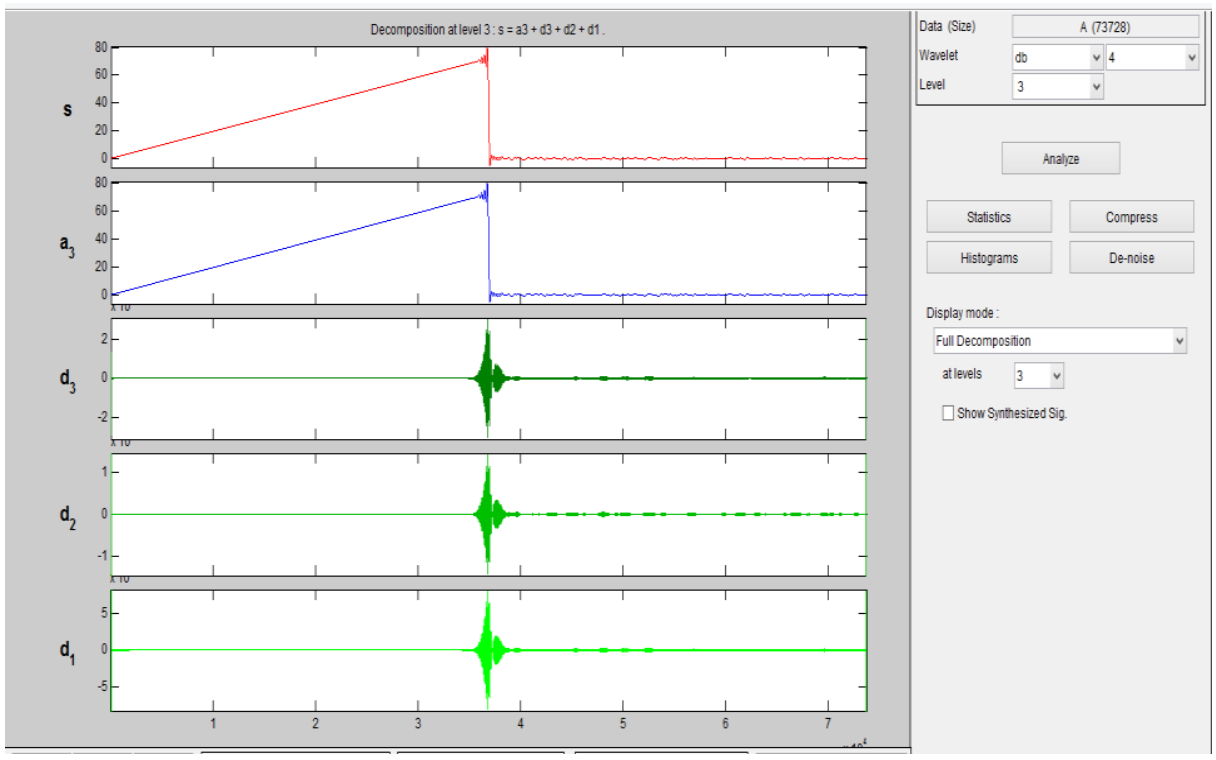

Figure 7. High-level Wavelet signals results from the DWT of experimental Balanced RK-4 rotor at a higher speed of $2570 \mathrm{rpm}$ by a db4 Wavelet.

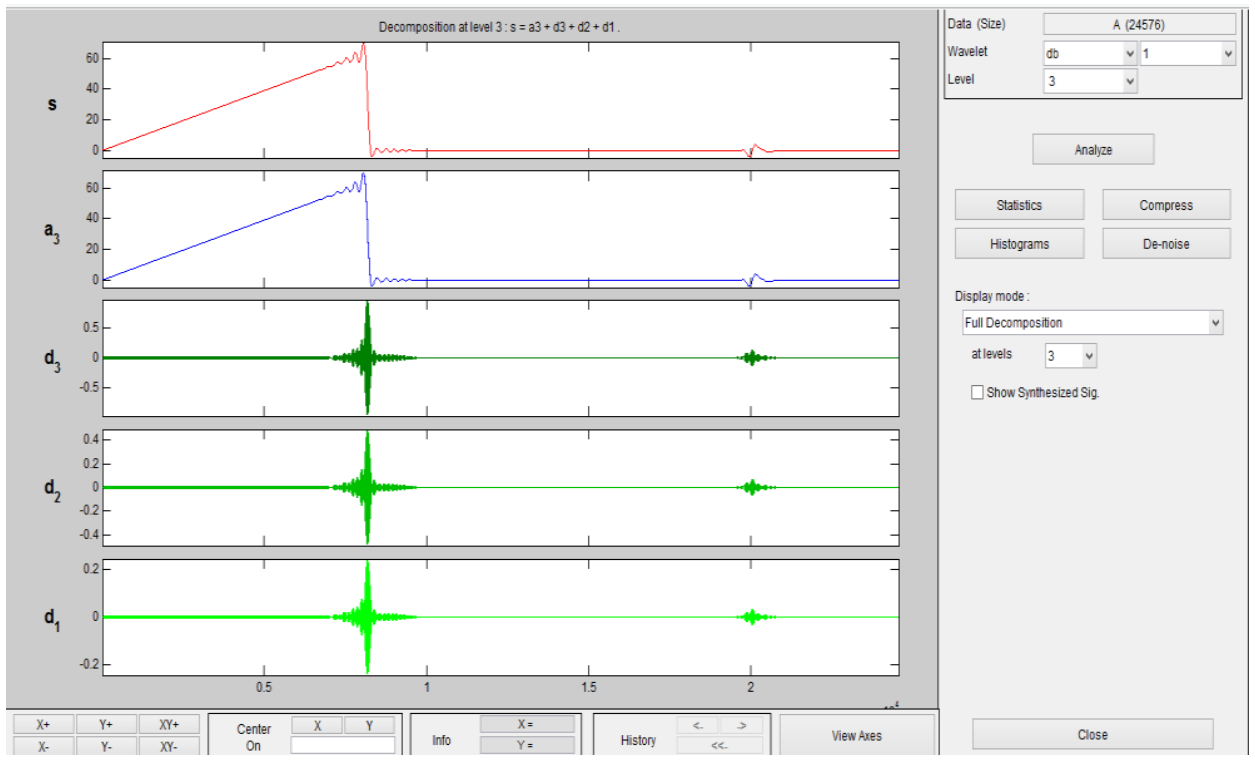

Figure 8. Signal Approximation and Decomposition of unbalanced RK-4 rotor at a speed of $2570 \mathrm{rpm}$ by a db1 Wavelet at level(s) $1,2,3$.

Using DWT, a multiresolution analysis of the vibration signals of the healthy rotor was also performed. The measured vibration signal was decomposed into three levels using Daubechies family as the mother wavelet. The vibration signal of the healthy rotor and the detail components of the 3 level decomposition are depicted in Fig. 7 and for unbalance effect in Fig. 8. The scaled signal values of detail coefficient $d 3$ obtained from the balanced rotor response at $2570 \mathrm{rpm}$ are shown in Figs. 7 and 8 . The results indicate that, the level-3 detail coefficient $d 3$ were more sensitive to the effect of faults. It was noted that, the balance alters the transient responses in the baseline, which can be detected by carefully comparing Figs. 7 and 8 . 
Comparison between the plots of DWT in Figs. 7 and 8 shows slight changes in response. In Fig.8, the main resonance peak is represented by a straight-line change in shape and magnitude (stretching the signal at the maximum and minimum frequency sub-bands), standard deviations were still observable at level $a 3$ with insignificant vibration which could be attributed to the centrifugal force. The 3rd detail-level plot showed a little difference between the two cases, the difference was significant to identify unbalance features. The $3 \mathrm{rd}$ level approximation (a3) response contains the dilated signal at a very low frequency and diagnoses rotor unbalance.

\subsection{Effect of unbalance and rub}

Evolution of this phenomenon at various motor speeds has been investigated in this section. The clearance was set at $\Delta=1.65 \times 10^{-6} \mathrm{~m}$ and experimental data on RK-4 was taken and analysis using FFT and DWT as observed and analysed using Figs. 9, 10, and 11.

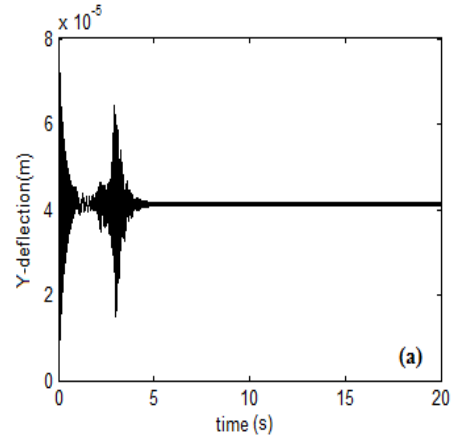

a) Vertical rotor deflection

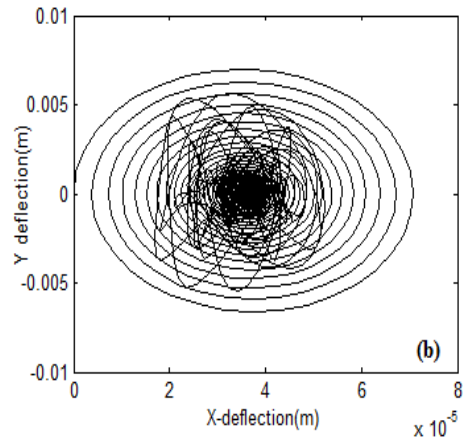

b) Rotor response orbit

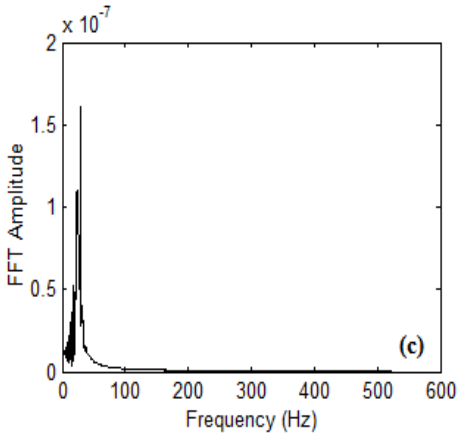

c) Y-Lateral frequency signal

Figure 9. Simulated rotor response of unbalanced rotor, with rub, axial force: $\Delta K / K_{b}=0, \psi=0$

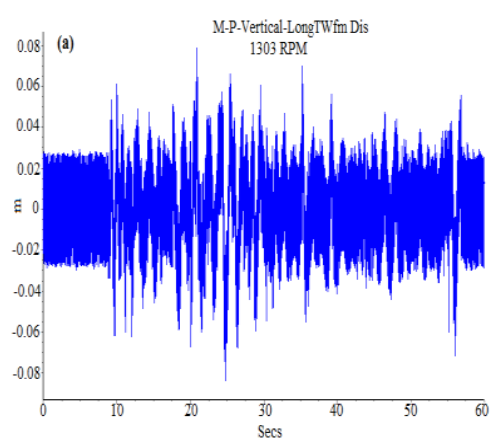

a) Vertical rotor-stator deflection

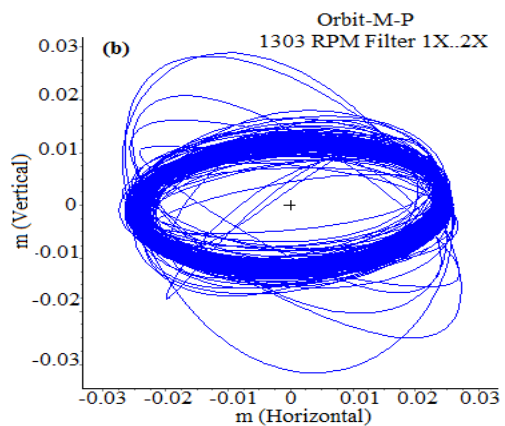

b) Rotor-stator response orbit

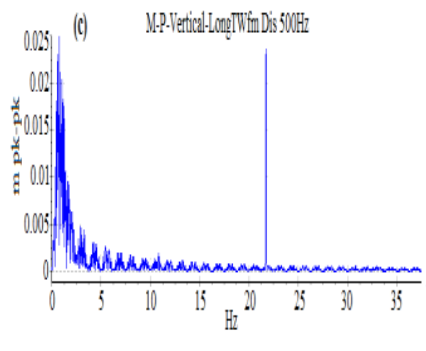

c) Shaft Frequency domain

Figure 10. Experimental unbalanced rotor system response of the RK.4 model at high speed $2570 \mathrm{rpm}$, with rotor-to-stator rub.

Fig. 9(a) displays the time-domain synchronous lateral response of the rotor. Critical speed of the rotor was found to be $799.6140 \mathrm{rpm}$ at $t=3 \mathrm{~s}$, a point at which the peak amplitude features in Fig. 9(a). The unbalance and reduction in clearance generated a large frictional force at each rotor-stator contact. Therefore, in Fig. 9, the amplitude of the peaks caused by unbalance and rub increased with the number of contacts. Alternatively, this observation could be explained by a decrease in clearance and the increased percentage of solid contact between the rotor and the stator. The experimental orbit in Fig. 10(b) shows a complex shape due to multiple rotor-stator rebounds at points of impact. A significant distortion in orbits due to rub in the system was obvious and correlates with the simulated orbit in Fig. 9(b). The inside circular-loop orbit observed for a case of unbalance was drastically modified to an outside elliptic shaped characteristic of severe rub in Fig. 10(b). Existence of 
considerable disparities in time-deflections under rub was noted with an increased level of vibration contacts in Fig. 10 (a) where the first critical speed was observed at $t=32 \mathrm{~s}$ experimentally and at $t=3 \mathrm{~s}$ by simulation in Fig. 9(a).

Experimentally it was noted that, rotor-stator rub increased the rotor critical speed to $1303 \mathrm{rpm}$. This is because rotor-stator contact increased the rotor dynamic stiffness, which in turn increased the rotor critical speed from $\omega l=1124.27 \mathrm{rpm}$ in Fig. 5 (c) to $\omega l=1102.43 \mathrm{rpm}$ in Fig. 10 (c). The experimental frequency spectrum in Fig. 10 (c) indicates an increased magnitude of frequency components as observed previously in Fig. 9 (c) by the appearance of squashed sub-harmonic peaks before the main peaks. It should be noted that, the tightening of $1 / 2 \mathrm{X}$ frequency peaks (sub-harmonics) in Fig. 10 (c) was mainly due to rub. In Fig. 10 (c), the main peak coincided with the rotor frequency at $22.5 \mathrm{~Hz}$. The gap between two consecutive peaks was in the range of $22.5 \mathrm{~Hz}$, which indeed was the shaft's rotation frequency.

Severity of rub is therefore a function of the number and the size of the various peaks observed in the scalogram. Based on the size of the inside loop, the abrupt change in frequency, and the variation in deflection waveform as the rotor traverse the first critical speed can be used as an indicator of rub and the bounces inside the orbit loops can be assigned as rub signature. The assigned rub signature has been experimentally used in the Discrete Wavelet analysis of wavelet coefficients to detect the occurrence of rotor-stator rub as illustrated in Fig. 11.

The results, presented in Fig. 11 revealed a series of shocks characterized by the lateral bands, spaced from the rotational frequency (about $7.88 \mathrm{~Hz}$ ) around a frequency of $588 \mathrm{~Hz}$ observed on the Scout 100 data acquisition screen. Further, unbalance features were observable in the approximated signal $a 2$ before contact occurred. The signal in Fig. 11 presented the characteristics of an unbalance, observed before the main peak of resonance represented by a straight line with greater magnitude and deviations observable at level $a 2$, and followed by several harmonics at lower amplitudes.

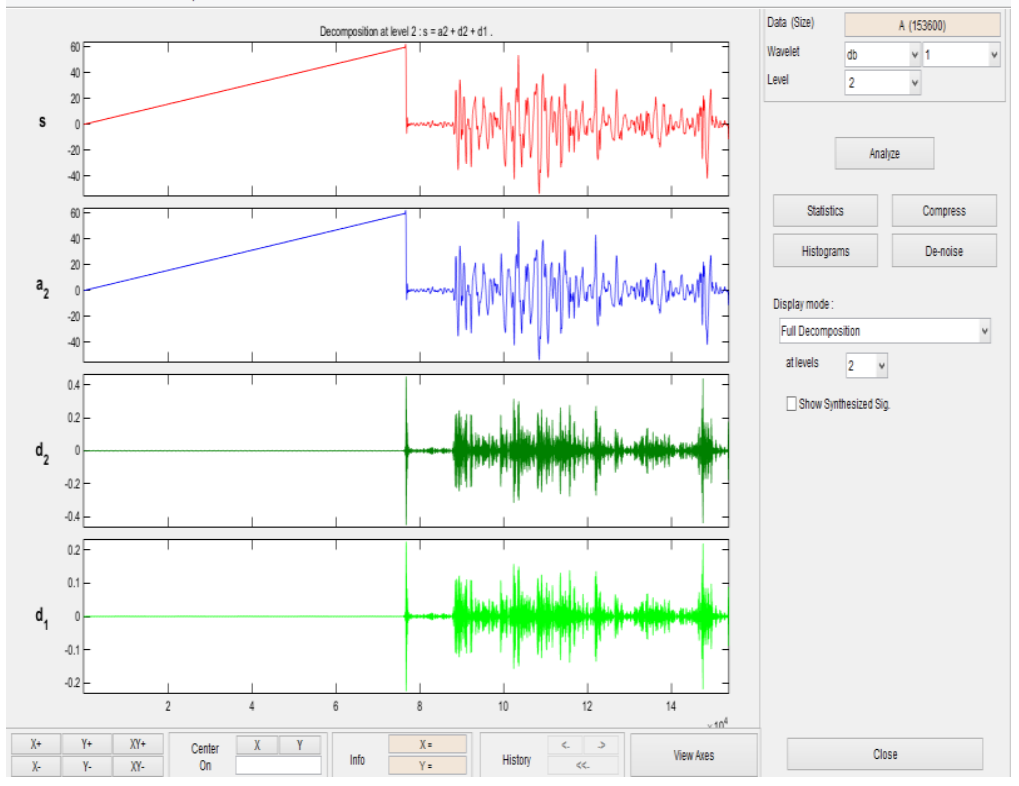

Figure 11. Two-level detail and approximate wavelet coefficients of unbalanced RK-4 rotor with rub at $2570 \mathrm{rpm}$ by a db1 wavelet at level(s) 1,2 .

\subsection{Excitation of unbalanced rotor by a crack}

The analysis was performed on an unbalanced rotor with a transverse crack. The responses were processed by FFT and DWT. The system response with a crack gave the orbit and the amplitude spectrum in Figs. 12, 13 and 14. 


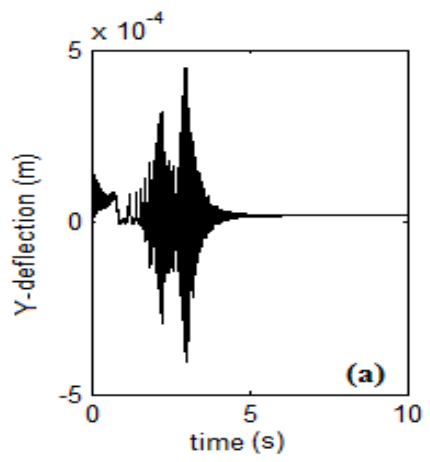

a) Y- cracked rotor deflection

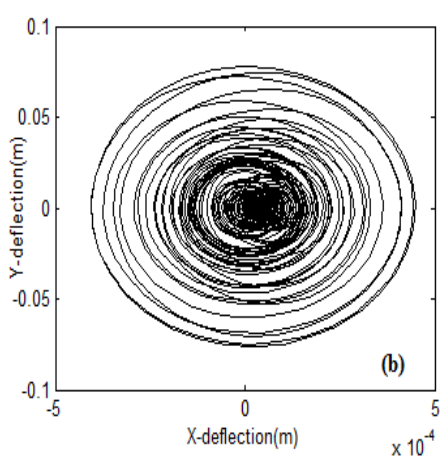

b) Cracked rotor response orbit

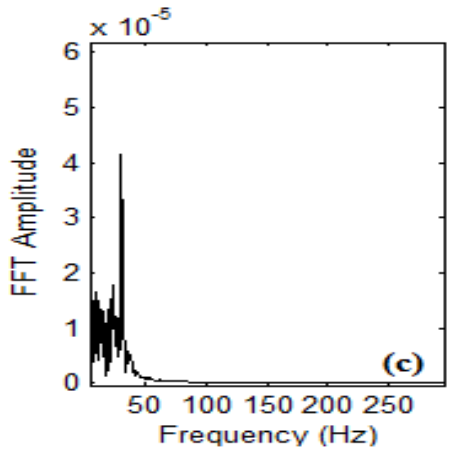

c) Y-Lateral frequency

Figure 12. Simulated dynamic response of cracked rotor via the critical speed, with axial force, and no rub.

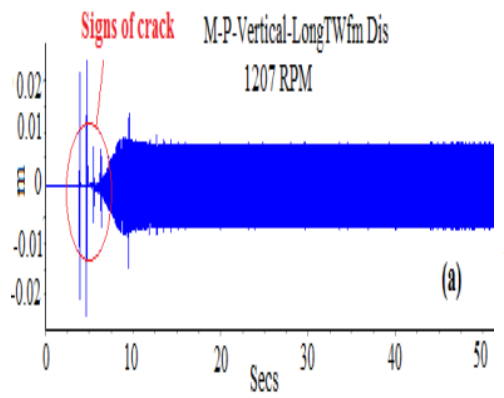

a) Vertical cracked rotor deflection

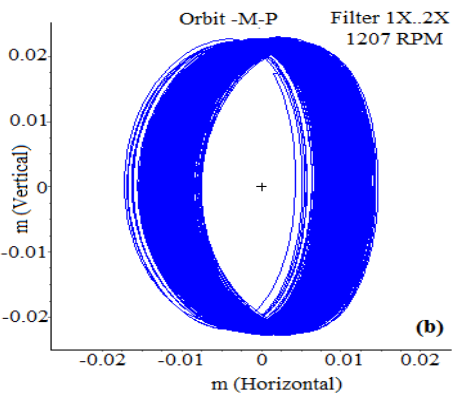

b) Experimental cracked rotor orbit

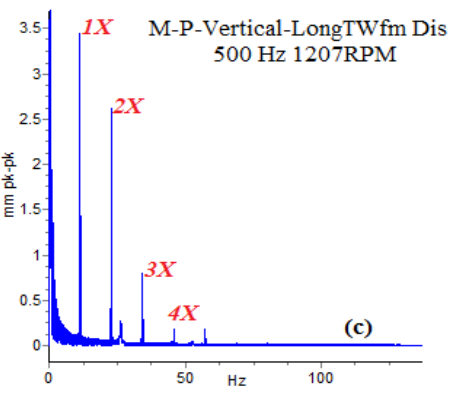

c) Frequency signal domain

Figure 13. Experimental rotor system response of the RK.4 model at $2570 \mathrm{rpm}$, with unbalance and crack $\left(\Delta K / K_{b}=0.0542\right)$.

Comparing the simulated orbits in Figs. 12 (b) and 5(b), it was apparent that, the breathing crack caused extra evolutions in the vibration response near the system critical speed. The maximum orbit amplitudes in Fig. 5 (b) were modified from $R_{1}=3.167 \times 10^{-6} \mathrm{~m}$ to $R_{2}=5.03 \times 10^{-4} \mathrm{~m}$ when the speed varied respectively, from $\omega l=1132.27 \mathrm{rpm}$ in Fig. 5 (c) to $\omega 1=1855.43 \mathrm{rpm}$ in Fig. 12 (c), i.e, from an uncracked to a cracked shaft.

The presence of a crack indicated strong tightening loops in Fig. 12 and 13 (b). In Fig. 13 (c), a high vibrational level was attained by order $1 X$ harmonic at $23.46 \mathrm{~Hz}$. At this point, the crack generated a tremendous increase in vibration amplitudes, characterized by the $2 X$ and $3 X$ super harmonic peaks. From the deflection in Figs. 12 (a) and 13 (a), crack features were manifested by small tight peaks at the rotor start-up, unlike the observations in the previous analysis. In spite of crack features, unbalance characteristic could not be well discriminated. Therefore, DWT was performed and the corresponding responses are shown in Fig. 13.

An analysis of the decomposed and reconstructed response indicated that, the vibration amplitudes were significant in the three lowest-frequency bands, $d 1, d 2$, and $d 3$. The presence of the peak linked to the second sub-critical speed appears more evident in the $d 3$ analysis than the approximated signal $a 3$. The spectrums of a crack fault obtained by using db23 reveal the presence of two prominent peaks corresponding to $1 X$ and $2 X$ harmonics, at two different times respectively, $(t=9500 \mathrm{~s}$ and $t=13880 \mathrm{~s})$ in Fig. 14. The effects of a crack generated a huge increase in vibration amplitude as previously observed in 2-D deflection in Figs. 12 (a) and 13 (a). The vibration amplitude of $(1 X)$ harmonic at $t=1000 \mathrm{~s}$ was slightly higher than that of $(2 X)$ harmonic at $t=14000 \mathrm{~s}$, until passing the critical speed after which, the amplitude became less than the $1 X$ harmonic. These observations are a unique indicator and are in agreement with the results obtained in [10]. 


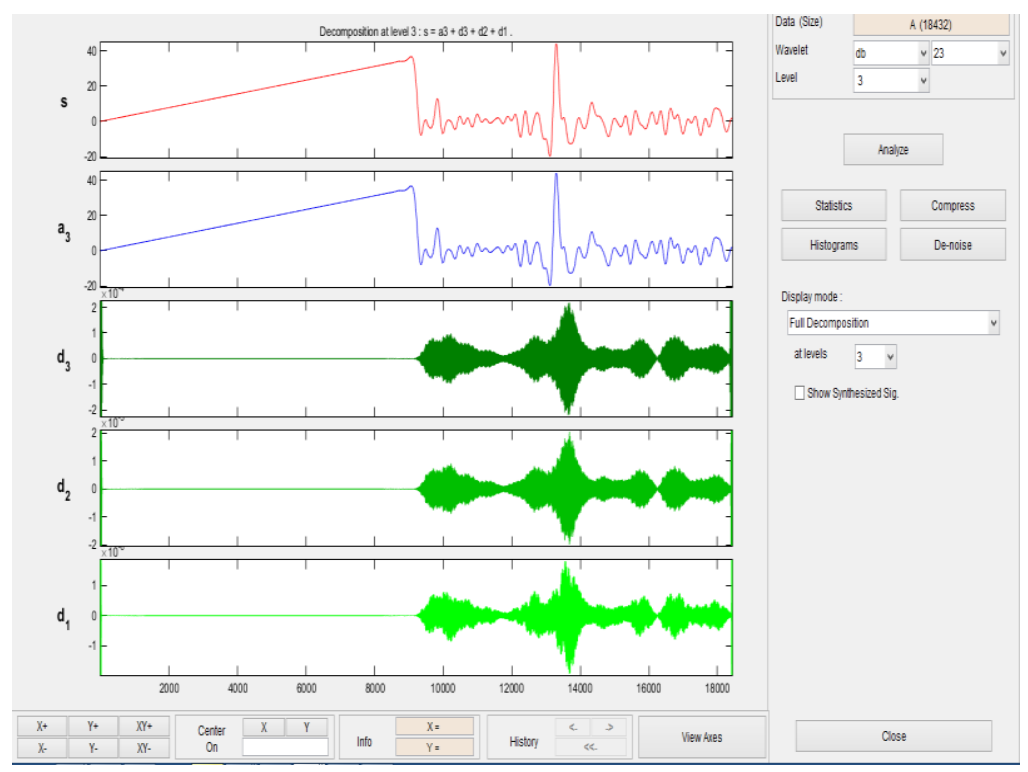

Figure 14. Signal Approximation and Decomposition of unbalanced RK-4 rotor with crack at 2570 rpm by a $\mathrm{db} 23$ Wavelet at level(s) 1, 2, 3 .

\subsection{Excitation of unbalanced rotor by a crack and rub}

In this section, the vibration of the unbalanced rotor-stator system at a high speed under rub and a breathing crack was analyzed by simulation and experiment. The system responses are presented in the Figs. 15, 16 and 17.

In Figs. 15 (a) and 15 (b), the crack and rub combined generated high vibration as indicated by vertical deflection and orbit patterns. Similar to the results in the previous section, unbalance and rub increased the rotor dynamic stiffness, which in turn increased the rotor critical speed. However, the crack increased shaft flexibility and decreased the critical speed of the rotor as observed in Fig. 15 (a). The harmonic resonances $1 X, 2 X, 3 X$ and $1 / 2 X$ were observed in the FFT in Fig. 15 (c), $1 X$ were excited by unbalance, whereas the $2 X, 3 X$ (super-harmonic) occurred due to the crack, and the $1 / 2 X$ were excited by rub, with a maximum limit on frequency at $500 \mathrm{~Hz}$. The RK-4 speed became extremely unstable and in the range $1207-2570 \mathrm{rpm}$, it was not feasible to experimentally acquire measurements. In Fig. 16, the response was characterized by a main peak at the synchronous frequency of $22.5 \mathrm{~Hz}$. Abnormal movements of the shaft in the bearing can be detected using orbit plots, complicated motions, such as quasi-periodic even chaotic vibrations, that are observed under multi-faults operating conditions in Fig. 16(b). It was noted in Fig. 16 (c) that, for a given crack depth, unbalance excited the $1 X$ sup-harmonic resonance, whereas rub excited the $1 / 2 X$ and $1 / 3 X$ subharmonic resonance. Finally, it can be inferred that, the emergence of the sub-harmonics, $2 X, 3 X$ provide evidence on existence of a breathing crack. These can be easily distinguished near the first critical speed. This inference concurs with the results in [18].

In Fig. 16 (c), the amplitudes of the sub-harmonic and sup-harmonic resonance increased due to the interaction of rub, a breathing crack and unbalance at a high speed. The crack and noise induced the sup-harmonics, $2 X$ and $3 X$ in Fig. 16(c), which were of smaller magnitude compared to the $1 X$ harmonic. The trend in Fig. 16 (c) suggests that, the harmonics were sensitive to the rotor rotation speed. The results in Fig. 16 indicate that, the effect of the crack on the rotor response was considerably larger than that of rub as the amplitude of the later is considerably attenuated in comparison to that of the former. Once again, the experimental results correlated well with the simulated results in Fig. 15. Analysis of experimental test data revealed that, an unbalanced rotor with rub and a breathing crack could be identified by observing the evolution of the sub-harmonic and sup- 
harmonic frequencies; of $1 / 2 X$ and $2 X, 3 X$ near the critical speed region of $1 X$. However, due to high non-linearity the evolution of the orbit patterns and the associated noise peaks became considerably complex for combined fault identification.

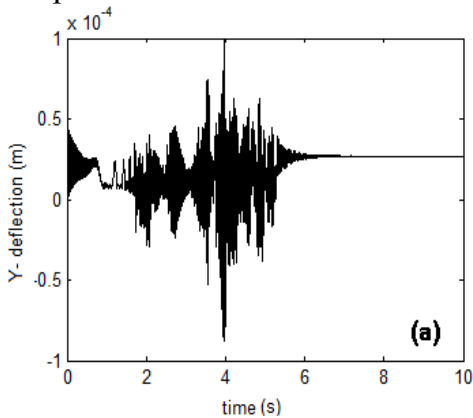

a) Multi-faults rotor deflection

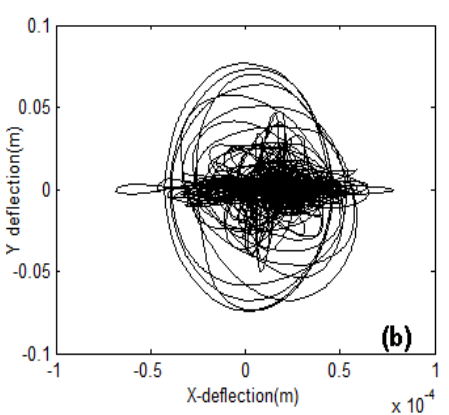

b) Multi-faults rotor orbit

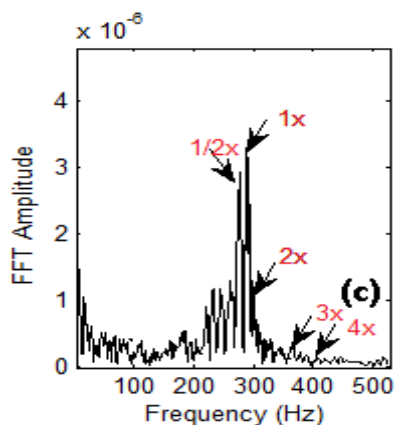

c) Y-Lateral frequency signal

Figure 15. Dynamic response of simulated unbalanced rotor model with rub, and crack, $\Delta K / K_{b}=0.0542$

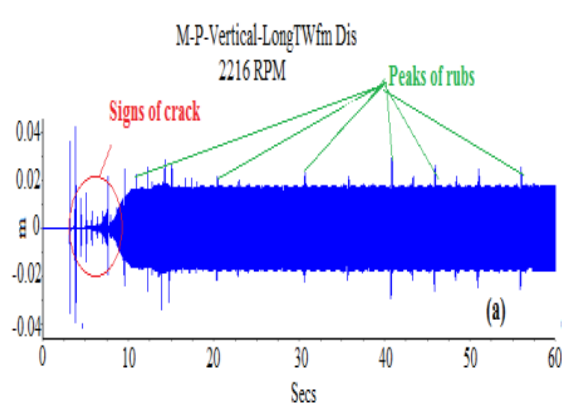

a) Unbalance-crack-rub RK-4 deflection

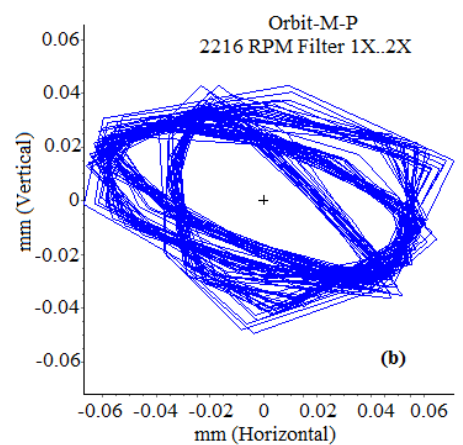

b) Experimental shaft orbit

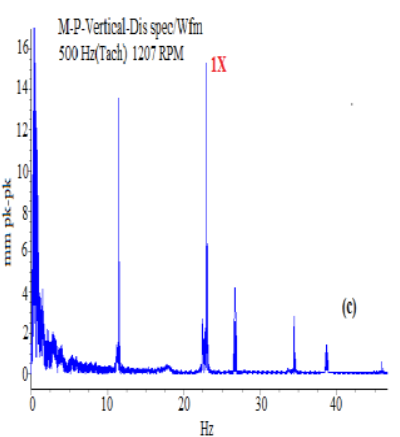

c) Frequency signal domain

Figure 16. Experimental rotor system response of the unbalanced RK. 4 model at higher speed $2570 \mathrm{rpm}$ with rub and $\operatorname{crack}\left(\Delta K / K_{b}=0.0542\right)$.

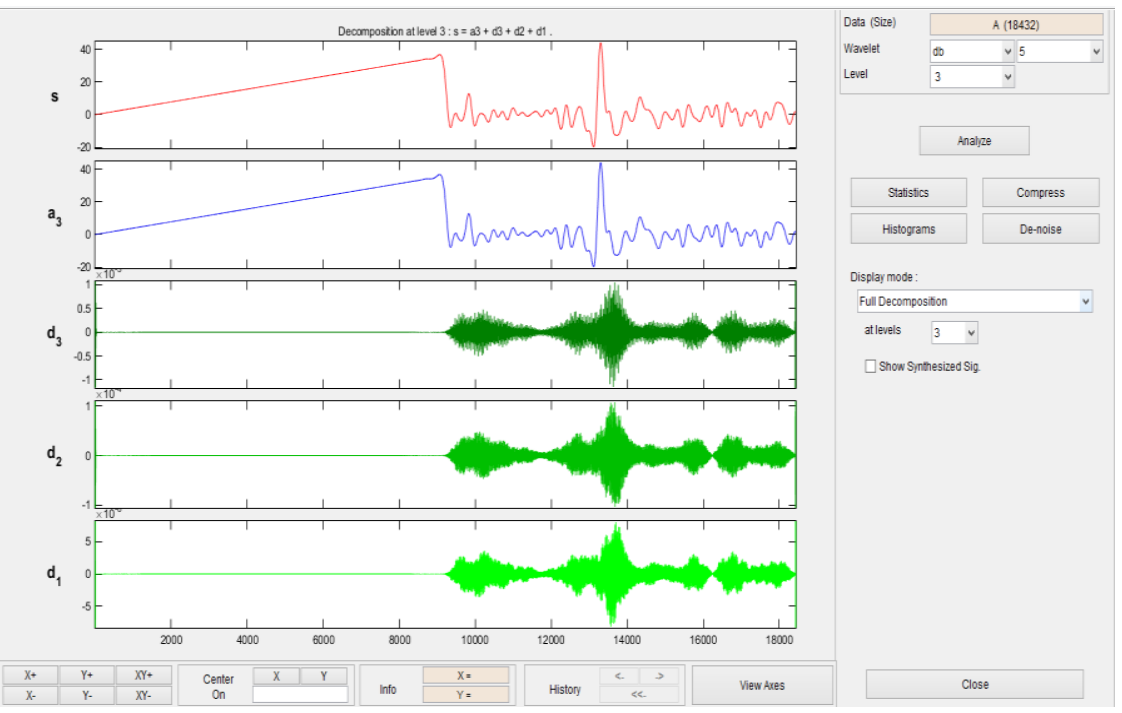

Figure 17. Signal Approximation and Decomposition of unbalanced RK-4 rotor with rub and crack at a speed $2570 \mathrm{rpm}$ by a db5 Wavelet at level(s) 1, 2, 3 . 
For further exploration, the system was accelerated to a higher speed of $2570 \mathrm{rpm}$, and held constant. The response near the rotor critical speed analyzed using DWT is illustrated in Fig. 17.

The multiresolution analysis applied to the signal using the $\mathrm{db} 5$ at level 3 decomposition extracted the detail and the approximation coefficient of vibrated RK-4 system. A qualitative difference in this case is apparent in the Fig.17. The result revealed as found previously, the features of the faults namely, unbalance, a breathing crack, and rub. The consistency of the experimental results presented so far, using DWT at various levels was manifested again in the Fig. 17, with the sole difference being that, the signal in Fig. 17 presented the noise feature due to contact.

From the results in Fig.17, it was observed that the measured signals were corrupted by multiple peak well observed in $(d 3)$ which is attributed to rub-impact. These vibration signals contained useful information in detecting the multiple faults. The unbalance and crack features are dominant in Fig. 17 at level $a 3$ approximation and the rub-impact characteristics are identifiable in $d 3$ decomposition. In summary, it was logical to conclude that sensitivity in the vibration tests could be enhanced by using Wavelet analysis. The latter provides a considerable diagnosis and an efficient method for multiple faults analysis.

\section{Conclusion}

This paper has presented a method for online multi-faults diagnosis; rub, a transverse breathing crack on unbalanced rotor-stator system. An analytical model of an extended Jeffcott rotor based on Energy Principles was used. Dynamics of a "breathing" crack was integrated into the model using Mayes steering function and rub was generated numerically using elastic impact-contact at the rub contact point. A unique pattern of vibration signals acquired from multiple probes mounted at various positions of the shaft allowed practical extraction and discrimination of features of mixed faults from the system response. Simulation strategies were applied to predict and discriminate the coexistence of multi-fault characteristics for validation. It was observed that, at a high rotating speed when a crack and rub coexist, the response becomes very complex to analyse using FFT. Multiresolution decomposition by DWT was performed in the form of discrete-time approximate and detail wavelet coefficients, respectively to break the measured unfaulty and multi-faulted system signals, into detailed versions. The DWT analysis using the Daubechies family demonstrated that, the magnitude of level three detail wavelet coefficient was sensitive to faults diagnostics and provides good discretisation signature from the original rotor signal. The effectiveness of the adopted method for multiple fault diagnosis has been demonstrated by the analysis of approximated and decomposed response signal. Modal parameters such as shaft deflection, orbit patterns and natural frequencies obtained from simulation results were sensitive to rub-impact and crack. The differences in simulated shaft deflection, orbit, by FFT and DWT analysis at higher speed reveal unique patterns of the various faults. The simulated and experimental results are well correlated. The simulated and experimental analysis demonstrated the consistency of the proposed method in the multi-faults identification by DWT in a real system. The DWT accurately discriminated unbalance, rub and crack symptoms in the rotating system.

\section{Acknowledgment}

Support by the Department of Mechanical Engineering, VUT towards this work is highly acknowledged by the authors.

\section{References}

1. T.H. Patel and A.K. Darpe, Journal of Sound and Vibration, 326(3), 740-752 (2009)

2. T. Zhou, J. Xu and W. Han, Journal of dynamic systems, measurement, and control, 127(3), 313$320(2005)$ 
3. N. Bachschmid and P. Pennacchi, In IMechE 7th Int. Conf. on Vibrations in Rotating Machinery (571-580, 2000)

4. A.K. Darpe, Dynamics of cracked rotor, PhD Thesis, IIT Delhi (2000)

5. T.H. Patel and A.K. Darpe, Journal of Sound and Vibration, 317(3), 841-865 (2008)

6. P. Flandrin, Time-frequency/Time-scale analysis, Academic Press (1999)

7. H. Kantz and T. Schreiber, Nonlinear time series analysis (Vol. 7). Cambridge university press (2004)

8. F. Al-badour, M. Sunar and L. Cheded, Mechanical Systems and Signal Processing, 25, 2083$2101(2011)$

9. C.S. Burrus, R.A. Gopinath and H. Guo, Introduction to wavelets and wavelet transforms: $a$ primer (Vol. 1, New Jersey: Prentice hall, 1998)

10. S. Prabhakar, A.S. Sekhar and A.R. Mohanty, Mechanical Systems and Signal Processing, 15(2), 447-450 (2001)

11. S.A. Adewusi and B.O. Al-Bedoor, Journal of sound and vibration, 246(5), 777-793 (2001)

12. T. Önsay and A.G. Haddow, The Journal of the Acoustical Society of America, 95(3), 1441-1449 (1994)

13. B.O. Al-Bedoor, Journal of Sound and vibration, 229(3), 627-645 (2000)

14. R. Sukkar and A.S. Yigit, Kuwait J. Sci. Eng, 35(2B), 143-170 (2008)

15. A.S. Yigit and A.P. Christoforou, Journal of sound and vibration, 234(1), 67-83 (2000)

16. A.D. Dimarogonas, Engineering fracture mechanics, 55(5), 831-857 (1996)

17. M. Misiti, Y. Misiti, G. Oppenheim and J. Poggi, Wavelet Toolbox for Use with MATLAB: User's Guide, The MathWorks, Natick, NA (2000)

18. J.J. Sinou, A. Loyer, O. Chiello, G. Mogenier, X. Lorang, F. Cocheteux and S. Bellaj, Journal of Sound and Vibration, 332(20), 5068-5085 (2013)

19. Z. Feng, M. Liang and F. Chu, Mechanical Systems and Signal Processing, 38(1), 165-205 (2013)

20. J. Zou and J. Chen, Journal of Sound and Vibration 276(1-2), 1-11 (2004) 